\title{
Suplementos orais artesanais desenvolvidos para pacientes com câncer: análise descritiva
}

\author{
Homemade oral supplements for patients \\ with cancer: descriptive analysis
}

Adriana GARÓFOLO'

Fernanda Rodrigues ALVES²

Maria Aurélia do Carmo REZENDE ${ }^{3}$

\section{R E S U M O}

\section{Objetivo}

Descrever a elaboração de oito formulações de suplementos artesanais orais desenvolvidos para aumentar o consumo de energia, proteínas e micronutrientes de pacientes com câncer, analisar seu valor nutricional e avaliar a apreciação do sabor, testando dois tipos de lipídeos.

\section{Métodos}

Os suplementos foram desenvolvidos com base em quatro ingredientes alimentares: leite, ovos, açúcares e óleos para recuperação nutricional. As formulações foram calculadas pelo programa de apoio à nutrição NUTWIN e seu valor nutricional foi comparado às recomendações para pacientes com câncer para macronutrientes e às Ingestões Diárias Recomendadas para micronutrientes. Por meio de degustação, os suplementos foram testados para verificação do sabor quando preparados com óleo ou margarina.

\section{Resultados}

A quantidade de energia por mililitro variou de 1,35 a 2,17kcal, tendo $39 \%$ a $59 \%$ de carboidrato, $11 \%$ a 13\% de proteína e 30\% a 49\% de lipídeo, fornecendo em média 43\% e 77\% da recomendação de energia e proteína, respectivamente. Vitaminas C e K, ácido fólico e manganês apresentaram 15\% de adequação em relação às recomendações. Com relação ao sabor, 78\% dos pacientes que experimentaram com óleo e 85\% dos que experimentaram com margarina relataram sabor bom, sem diferença estatística entre os tipos de suplementos.

\footnotetext{
1 IAG - Assistência, Ensino e Pesquisa. R. Brigadeiro Luis Antonio, 2729, Conj. 805, Paraíso, 01401-000, São Paulo, SP, Brasil. Correspondência para/Correspondence to: A. GARÓFOLO. E-mail: <adrigarofolo@hotmail.com>.

${ }^{2}$ Hospital Samaritano de São Paulo, Setor de Oncologia e Hematologia. São Paulo, SP, Brasil.

3 Instituto de Oncologia Pediátrica. São Paulo, SP, Brasil.
} 
524 | A. GARÓFOLO et al.

\section{Conclusão}

A avaliação do sabor demonstrou que a maioria dos pacientes considerou o suplemento com sabor bom. Essas taxas foram superiores quando testados com margarina. Os resultados sugerem que o uso de suplementos orais artesanais pode ser uma alternativa viável em situações onde não há recursos suficientes para aquisição dos industrializados.

Termos de indexação: Estado nutricional. Neoplasias. Nutrição. Suplementos dietéticos. Terapia nutricional.

\section{A B S T R A C T}

\section{Objective}

This study aimed to describe the development of eight formulations of homemade oral supplements that propose to increase the energy, protein and micronutrient intakes of patients with cancer, analyze its nutritional value and assess its taste using two different fat sources.

\section{Methods}

The supplements were based on four ingredients: milk, eggs, sugars and oils for nutritional recovery. The formulations were calculated by the nutritional support software NUTWIN. The nutritional value of the formulations was compared with the recommendations for cancer patients for macronutrients and with the Recommended Daily Intakes for micronutrients. The supplements were tested. The supplements underwent taste tests to determine if the patients preferred supplements prepared with oil or margarine.

\section{Results}

The amount of energy per milliliter varied from 1.35 to $2.17 \mathrm{kcal}$. The carbohydrate content varied from $39 \%$ to $59 \%$, protein content from $11 \%$ to $13 \%$ and fat content from $30 \%$ to $49 \%$, providing roughly $43 \%$ and $77 \%$ of the recommended energy and protein intake, respectively. The contents of vitamins C and K, folic acid and manganese represented $15 \%$ of the recommended daily intakes. More patients approved the taste of the supplements prepared with margarine (85\%) than with oil (78\%) but the difference was not significant.

\section{Conclusion}

Taste tests showed that most patients liked the taste of the supplements. Supplements prepared with margarine had better acceptance. The results suggest that the use of homemade oral supplements can be a viable alternative for people who do not have the resources to buy commercial oral supplements.

Indexing terms: Nutritional status. Neoplasms. Nutrition. Dietary supplements. Nutritional therapy.

\section{N T R O D U Ç Ã O}

O câncer infanto-juvenil compreende 0,5\% a 3,0\% de todas as neoplasias malignas humanas na maioria das populações ${ }^{1}$, com incidência anual de aproximadamente 200 mil casos em todo o mundo. Apesar disso, nos países desenvolvidos é a segunda maior causa de morte em crianças com menos de 15 anos, perdendo somente para os acidentes. Sua incidência é normalmente superior no sexo masculino².

Os tipos mais comuns de câncer entre crianças e adolescentes são as leucemias, seguidas pelos tumores cerebrais, linfomas, neuroblastomas, sarcomas de partes moles, tumor de Wilms, tumores ósseos e retinoblastoma ${ }^{3}$. É uma doença catabólica, em que o tumor maligno atua de forma a consumir as reservas nutricionais do hospedeiro, levando ao prejuízo nutricional. Entretanto, alguns tumores estão mais associados com a desnutrição, principalmente os tumores sólidos, provavelmente pelo crescimento mais indolente ${ }^{4,5}$.

As alterações nutricionais podem ocorrer em decorrência do tratamento, sendo a desnutrição o distúrbio mais importante. Geralmente está associada à intensidade da terapia antineoplásica, que causa efeitos negativos sobre a função gastrintestinal, além de outros efeitos tóxi$\cos$. Os agentes quimioterápicos utilizados em altas doses, comumente, induzem a náusea e vômitos intensos, diarreia, constipação, má absorção de nutrientes intestinais e mucosites. Além disso, 
pode ocorrer dor no local do tumor que, indiretamente, pode interferir com a alimentação6,7.

A importância da terapia nutricional na criança com câncer está baseada na ideia de que o funcionamento dos sistemas orgânicos vitais é mantido mais adequadamente quando o estado nutricional do paciente está preservado. Em situações de desnutrição grave, adaptações fisiológicas preservam a vida de indivíduos desnutridos, porém na criança desnutrida com câncer os sistemas também são afetados pela terapia. Portanto, o metabolismo dos agentes quimioterápicos e dos nutrientes pode estar comprometido nessa situação ${ }^{8,9}$. Crianças com câncer apresentam comprometimento imunológico como efeito da quimioterapia ou radioterapia em medula óssea, devido à supressão medular. Como a desnutrição também causa prejuízos na função imune, indivíduos desnutridos com câncer têm aumento no risco de adquirir infecções e, portanto, maior morbidade do que os eutróficos ${ }^{10-13}$.

O estado nutricional de pacientes pediátricos com câncer se correlaciona com múltiplos fatores, atuando em maior ou menor intensidade e determinando riscos diferentes para cada grupo ou indivíduo. Apesar das condições descritas, existem poucos estudos que avaliaram a terapia nutricional protocolada em crianças com câncer e nenhum relato sobre suas estratégias por via oral.

O objetivo deste estudo foi descrever a elaboração de oito formulações de suplementos artesanais hipercalóricos desenvolvidos para aumentar o fornecimento de energia, proteína e micronutrientes na dieta de pacientes com câncer.

\section{MÉ T O D O S}

Este artigo faz parte de um projeto maior que utiliza suplementos artesanais para terapia nutricional de pacientes com câncer em tratamento no Instituto de Oncologia Pediátrica da Universidade Federal de São Paulo, o qual obteve aprovação do Comitê de Ética Médica da mesma (CEP n. 1490/05).

Inicialmente foram elaborados os suplementos artesanais orais e na sequência aplicou- -se uma pesquisa de opinião. Participaram pacientes que vieram ao hospital para consulta ambulatorial e aqueles que estavam recebendo quimioterapia de ambulatório.

Oito tipos diferentes de Suplementos Orais Artesanais (SOA) foram desenvolvidos com base em cinco ingredientes alimentares: leite, ovo, açúcar, amido de milho e lipídeo (óleo ou margarina).

A criação destas preparações teve a finalidade de aumentar a oferta de energia e melhorar a oferta proteica e de micronutrientes. Depois da elaboração das formulações, foram calculados os valores nutricionais com a utilização do programa de apoio à nutrição NUTWIN ${ }^{14}$ da Universidade Federal de São Paulo.

\section{Valor nutricional dos suplementos artesanais}

1) Valor nutricional dos $S O A$ em relação às recomendações: os valores de energia e proteína foram comparados com as recomendações para pacientes pediátricos com câncer (Children's Oncology Group e Current Therapy and Guidelines from the Children's Cancer Group) ${ }^{15,16}$. Os micronutrientes foram comparados às recomendações das Dietary Recommended Intakes (DRI) ${ }^{17,18}$. Para essa análise comparativa, estimamos o consumo médio diário em mililitro, de acordo com as faixas etárias apresentadas nas recomendações. Os valores encontrados para cada faixa etária compuseram um volume médio de ingestão que foi de aproximadamente $535 \mathrm{~mL}$ por dia (Tabela 1).

2) Valor nutricional dos SOA em relação ao valor nutricional de suplementos industrializados: obtivemos a média do valor nutricional de cada nutriente das oito formulações, resultando em um valor nutricional médio dos suplementos artesanais. Para avaliar as porcentagens de adequação dos nutrientes existentes no SOA, utilizamos este valor nutricional médio encontrado nas preparações artesanais e comparamos com o valor existente em dois suplementos industrializados com formulação padrão (Nutren 1.0 e Jr ${ }^{\circledR}$ ). 
526 | A. GARÓFOLO et al.

Tabela 1. Composição nutricional dos suplementos artesanais em 100 mililitros. São Paulo (SP), 2005.

\begin{tabular}{|c|c|c|c|c|c|c|c|c|c|}
\hline \multirow{2}{*}{ Nutriente por $100 \mathrm{~mL}$} & \multirow{2}{*}{ Unidade } & \multicolumn{8}{|c|}{ Formulações } \\
\hline & & 1 & 2 & 3 & 4 & 5 & 6 & 7 & 8 \\
\hline \multirow[t]{2}{*}{ Energia } & kcal & 161,00 & 136,00 & 166,00 & 141,00 & 194,00 & 217,00 & 185,00 & 172,00 \\
\hline & g & 19,00 & 16,00 & 24,50 & 18,00 & 19,00 & 26,00 & 21,00 & 21,00 \\
\hline \multirow[t]{2}{*}{ Carboidrato } & $\%$ & 47,00 & 46,00 & 59,00 & 52,00 & 39,00 & 49,00 & 45,00 & 50,00 \\
\hline & $g$ & 5,00 & 4,50 & 4,50 & 4,00 & 6,00 & 6,00 & 5,50 & 5,50 \\
\hline \multirow[t]{2}{*}{ Proteína } & $\%$ & 13,00 & 13,00 & 11,00 & 12,00 & 12,00 & 11,00 & 12,00 & 13,00 \\
\hline & $g$ & 7,00 & 6,00 & 5,50 & 5,50 & 10,50 & 9,50 & 9,00 & 7,00 \\
\hline Lipídeo & $\%$ & 40,00 & 41,00 & 30,00 & 36,00 & 49,00 & 40,00 & 43,00 & 37,00 \\
\hline Cálcio & $\mathrm{mg}$ & 137,00 & 121,00 & 112,00 & 101,00 & 179,00 & 161,00 & 151,00 & 133,00 \\
\hline Ferro & $\mathrm{mg}$ & 0,60 & 0,07 & 0,90 & 0,10 & 0,30 & 0,30 & 0,10 & 0,07 \\
\hline Magnésio & $\mathrm{mg}$ & 7,70 & 7,50 & 7,30 & 12,30 & 7,50 & 6,70 & 9,80 & 7,50 \\
\hline Fósforo & $\mathrm{mg}$ & 103,20 & 91,00 & 83,00 & 77,60 & 160,70 & 144,70 & 224,50 & 100,60 \\
\hline Potássio & $\mathrm{mg}$ & 184,30 & 169,70 & 149,00 & 207,80 & 223,50 & 201,20 & 133,70 & 179,80 \\
\hline Sódio & $\mathrm{mg}$ & 81,90 & 72,30 & 71,00 & 83,40 & 81,70 & 123,40 & 95,30 & 126,70 \\
\hline Zinco & $\mathrm{mg}$ & 1,10 & 0,40 & 1,30 & 0,30 & 0,80 & 0,70 & 0,60 & 0,40 \\
\hline Cobre & mcg & 20,00 & 20,00 & 20,00 & 38,00 & 38,00 & 20,00 & 20,00 & 20,00 \\
\hline Manganês & mg & 0,01 & 0,01 & 0,01 & 0,40 & 0,01 & 0,00 & 0,01 & 0,01 \\
\hline Selênio & mcg & 5,50 & 4,90 & 5,00 & 4,60 & 7,50 & 6,70 & 1,30 & 5,40 \\
\hline Vitamina C & $\mathrm{mg}$ & 1,00 & 0,50 & 1,00 & 2,00 & 0,80 & 0,70 & 0,80 & 0,50 \\
\hline Vitamina $B_{1}$ & $\mathrm{mg}$ & 0,10 & 0,04 & 0,20 & 0,04 & 0,60 & 0,60 & 0,50 & 0,40 \\
\hline Vitamina $\mathrm{B}_{2}$ & $\mathrm{mg}$ & 0,40 & 0,20 & 0,40 & 0,20 & 0,30 & 0,30 & 0,30 & 0,30 \\
\hline Niacina & $\mathrm{mg}$ & 2,50 & 0,90 & 3,00 & 0,90 & 1,00 & 1,00 & 0,60 & 0,10 \\
\hline Ácido Pantotênico & $\mathrm{mg}$ & 0,40 & 0,30 & 0,30 & 0,30 & 0,70 & 0,60 & 0,50 & 0,40 \\
\hline Vitamina $\mathrm{B}_{6}$ & mg & 0,20 & 0,03 & 0,20 & 0,10 & 0,70 & 0,06 & 0,05 & 0,03 \\
\hline Ácido Fólico & mcg & 2,60 & 0,30 & 1,80 & 5,20 & 12,50 & 11,30 & 4,60 & 2,60 \\
\hline Vitamina $\mathrm{B}_{12}$ & mcg & 0,60 & 0,40 & 0,60 & 0,40 & 0,80 & 0,70 & 0,50 & 0,50 \\
\hline Vitamina A & RE & 81,20 & 71,40 & 29,70 & 69,60 & 134,10 & 120,70 & 102,40 & 79,20 \\
\hline Vitamina E & ATE & 1,70 & 0,80 & 2,00 & 0,80 & 1,20 & 1,10 & 0,60 & 0,90 \\
\hline Vitamina D & mcg & 0,80 & 0,70 & 0,60 & 0,50 & 1,20 & 1,10 & 0,70 & 0,80 \\
\hline Vitamina K & mcg & 0,20 & 0,20 & 5,00 & 0,30 & 0,30 & 0,30 & 0,30 & 0,20 \\
\hline Biotina & mcg & 3,00 & 2,60 & 2,50 & 2,70 & 5,50 & 4,90 & 3,50 & 2,90 \\
\hline
\end{tabular}

1: chocolate frio; 2: leite com café; 3: chocolate quente; 4: leite com banana; 5: mingau branco; 6: mingau com sabor; 7: leite com sorvete; 8: leite sabor morango.

\section{Avaliação do sabor}

Cada indivíduo degustou uma única vez cada preparação. No total houve 312 degustações durante 16 semanas consecutivas. Cada indivíduo degustou a preparação e em seguida respondeu a um questionário que continha perguntas fechadas sobre o sabor (bom ou ruim) e abertas para relato da opinião. Os suplementos foram preparados com dois tipos de lipídeos, sendo testados com a utilização de margarina ou de óleo vegetal de soja. A preferência das preparações com os diferentes tipos de lipídeos foi avaliada e os resultados foram comparados.
Para o teste sensorial, onde se avaliou o sabor das preparações, comparando entre os diferentes tipos de lipídeos foram utilizados, aplicamos o teste de Qui-quadrado.

RESULTADOSE DISCUSSÃO

\section{Composição e elaboração dos suplementos artesanais}

A terapia nutricional pode apresentar impacto positivo para o estado nutricional e funcionamento dos sistemas orgânicos vitais ${ }^{8,9}$. 
As formulações foram desenvolvidas com o objetivo de compor um suplemento com boa palatabilidade e com fornecimento de quantidades equilibradas de macronutrientes, além da maior oferta dos micronutrientes com a utilização de ingredientes dietéticos.

Os oito suplementos possuem como ingredientes base o açúcar, lipídeo, leite, ovo (clara ou gema) e amido de milho. Duas preparações são mingaus, uma delas utiliza sorvete na composição e resultando em uma preparação tipo milk shake e as demais são bebidas quentes ou frias com diversos sabores (Tabela 1).

\section{Valor nutricional dos suplementos artesanais}

A quantidade de energia por mililitro de suplemento variou de 1,35 a $2,17 \mathrm{kcal}$ com a seguinte distribuição: 39\% a 59\% de carboidrato, $11 \%$ a $13 \%$ de proteína e 30\% a 49\% de lipídeo. O valor nutricional de cada uma das oito preparações esta descrito na Tabela 2.

O valor nutricional dos suplementos artesanais foi comparado com o valor nutricional de um suplemento industrializado e com o valor das recomendações nutricionais (conforme descrito

Tabela 2. Porcentagem de adequação dos nutrientes dos Suplementos Orais Artesanais conforme recomendações*. São Paulo (SP), 2005.

\begin{tabular}{|c|c|c|c|c|c|c|c|c|}
\hline $\begin{array}{l}\text { Faixa etária } \\
\text { (anos) }\end{array}$ & $\begin{array}{l}1 \text { a } 3 \\
F \text { e M }\end{array}$ & $\begin{array}{l}4 \text { a } 6 \\
F \text { e M }\end{array}$ & 7 a 10 & 11 a $14 \mathrm{~F}$ & 15 a $18 \mathrm{~F}$ & 11 a $14 \mathrm{M}$ & 15 a $18 \mathrm{M}$ & Média \\
\hline Volume & $300 \mathrm{~mL}$ & $450 \mathrm{~mL}$ & $450 \mathrm{~mL}$ & $600 \mathrm{~mL}$ & $600 \mathrm{~mL}$ & $600 \mathrm{~mL}$ & $750 \mathrm{~mL}$ & $535,71 \mathrm{~mL}$ \\
\hline Energia (kcal) & 40 & 43 & 39 & 47 & 47 & 42 & 43 & 43 \\
\hline Proteína (g) & 95 & 95 & 82 & 66 & 69 & 68 & 65 & 77 \\
\hline Faixa etária (anos) & & $\begin{array}{l}1 \text { a } 3 \\
\text { F e M }\end{array}$ & $\begin{array}{l}4 \text { a } 8 \\
F \text { e M }\end{array}$ & 9 a $13 \mathrm{~F}$ & 14 a $18 \mathrm{~F}$ & 9 a $13 \mathrm{M}$ & 14 a $18 \mathrm{M}$ & Média \\
\hline Volume & & $300 \mathrm{~mL}$ & $450 \mathrm{~mL}$ & $600 \mathrm{~mL}$ & $600 \mathrm{~mL}$ & $600 \mathrm{~mL}$ & $750 \mathrm{~mL}$ & $535,71 \mathrm{~mL}$ \\
\hline Vitamina A (mcg RE) & & 88 & 99 & 88 & 76 & 88 & 73 & 85 \\
\hline Vitamina C (mg) & & 20 & 18 & 13 & 9,0 & 13 & 10 & 14 \\
\hline Vitamina D (mcg) & & 50 & 76 & 101 & 101 & 101 & 126 & 92 \\
\hline Vitamina K (mcg) & & 9,6 & 7,9 & 9,6 & 7,7 & 9,6 & 9,6 & 9,0 \\
\hline Tiamina (mg) & & 216 & 270 & 240 & 216 & 240 & 225 & 235 \\
\hline Riboflavina (mg) & & 186 & 233 & 207 & 186 & 207 & 179 & 199 \\
\hline Niacina $(\mathrm{mg})$ & & 73 & 82 & 73 & 62 & 73 & 68 & 72 \\
\hline Vitamina $B_{6}(\mathrm{mg})$ & & 120 & 150 & 120 & 100 & 120 & 115 & 121 \\
\hline Folato $(\mathrm{mcg})$ & & 6,4 & 7,2 & 6,4 & 4,8 & 6,4 & 6,0 & 6,2 \\
\hline Vitamina $B_{12}(\mathrm{mcg})$ & & 197 & 222 & 197 & 148 & 197 & 184 & 191 \\
\hline Ácido pantotênico (mg) & & 69 & 69 & 69 & 55 & 69 & 69 & 67 \\
\hline Biotina $(\mathrm{mcg})$ & & 135 & 135 & 108 & 86 & 108 & 108 & 113 \\
\hline Vitamina E (mg ATE) & & 62 & 79 & 67 & 49 & 67 & 62 & 64 \\
\hline Cálcio (mg) & & 82 & 77 & 63 & 63 & 63 & 79 & 71 \\
\hline Magnésio (mg) & & 32 & 29 & 21 & 14 & 21 & 15 & 22 \\
\hline Fósforo (mg) & & 83 & 115 & 61 & 61 & 61 & 77 & 77 \\
\hline Selênio (mcg) & & 77 & 77 & 77 & 56 & 77 & 70 & 72 \\
\hline Zinco (mg) & & 76 & 68 & 57 & 51 & 57 & 52 & 60 \\
\hline Sódio (mg) & & 28 & 36 & 38 & 38 & 38 & 47 & 38 \\
\hline Potássio (mg) & & 18 & 22 & 24 & 23 & 24 & 29 & 24 \\
\hline Cobre (mcg) & & 22 & 25 & 21 & 17 & 21 & 21 & 21 \\
\hline Manganês (mg) & & 1,5 & 1,8 & 2,3 & 2,3 & 1,9 & 2,1 & 2,0 \\
\hline Ferro (mg) & & 15 & 15 & 26 & 14 & 26 & 23 & 20 \\
\hline
\end{tabular}

F: sexo feminino; M: sexo masculino.

Os nutrientes destacados em cor cinza foram aqueles que apresentaram média de adequação criticamente baixa em relação às recomendações. ${ }^{*}$ Recomendações de energia e proteína ${ }^{15,16}$ que propõem os valores da Recommended Dietary Allowances ${ }^{18}$ e recomendações de micronutrientes: $(\text { IDR })^{17}$. 
no delineamento). Comparando-se o valor nutricional oferecido pelos suplementos com as recomendações, observou-se que a proteína e 11 dos 23 micronutrientes estudados atingiram em média $70 \%$ ou mais da recomendação. Os micronutrientes com tal percentual foram: vitamina $A$, vitamina $D$, tiamina, riboflavina, niacina, vitamina $\mathrm{B}_{6}$, vitamina $\mathrm{B}_{12}$, biotina, cálcio, fósforo e selênio Os valores de energia foram aquém, entretanto com importante contribuição (43\%) na porcentagem total da dieta (Tabela 2).

A vitamina C e K, o ácido fólico e o manganês foram os nutrientes que se apresentaram criticamente baixos, ou seja, seus valores foram inferiores a $15 \%$ da recomendação média. Entretanto, tiamina, riboflavina, vitamina $B_{12}$ e biotina apresentaram médias percentuais de adequação acima da recomendação (Tabela 2).

A comparação do SOA com o Suplemento Industrializado (SI) mostrou que as vitaminas C e $\mathrm{K}$, ácido fólico, cobre e ferro foram os nutrientes que apresentam maior desvantagem quantitativa no SOA (Tabela 3).

Os suplementos industrializados podem auxiliar na terapia nutricional, porém seu alto custo dificulta sua aquisição, limitando o uso generalizado. Por outro lado, os suplementos artesanais, obtidos pela modulação artesanal de ingredientes dietéticos, podem ser uma opção menos onerosa que os suplementos industrializados. Apesar disso, existem algumas desvantagens quando comparados aos industrializados, como o menor controle microbiológico devido a maior manipulação. Também há menor praticidade, além da dificuldade em garantir valor nutricional equiparável, conforme visto nos resultados acima descritos.

Não há regulamentação específica acerca dos suplementos artesanais, entretanto, entendemos que as normatizações existentes para todo o conjunto de suplementos nutricionais possam, também, abranger os artesanais. Em 1994, nos Estados Unidos foi aprovado o Dietarry Supplement Health and Education Act (DSHEA) que apresenta a Lei da Saúde e Educação sobre Suplementos
Dietéticos. Esta lei classifica os suplementos nutricionais como produto alimentício acrescido à dieta com o objetivo de suplementação e que estes contenham vitamina, mineral, aminoácidos e outras substâncias dietéticas capazes de aumentar o conteúdo energético total da dieta ${ }^{19}$.

No Brasil a Agencia Nacional de Vigilância Sanitária (ANVISA) é o órgão responsável pela nor-

Tabela 3. Porcentagem média de adequação dos nutrientes dos suplementos artesanais e industrializados conforme as recomendações* e comparação percentual entre SOA e SI. São Paulo (SP), 2005.

\begin{tabular}{|c|c|c|c|}
\hline Nutriente & $\mathrm{SI} \%$ & SOA \% & SOASI \% \\
\hline Energia (kcal) & 25 & 43 & 172 \\
\hline Proteína (g) & 43 & 77 & 179 \\
\hline Vitamina A (mcg RE) & 94 & 85 & 91 \\
\hline Vitamina C (mg) & 154 & 14 & 9 \\
\hline Vitamina D (mcg) & 94 & 92 & 99 \\
\hline Vitamina K (mcg) & 42 & 9 & 21 \\
\hline Tiamina (mg) & 85 & 235 & 277 \\
\hline Riboflavina (mg) & 90 & 199 & 221 \\
\hline Niacina $(\mathrm{mg})$ & 84 & 72 & 85 \\
\hline Vitamina $\mathrm{B}_{6}(\mathrm{mg})$ & 72 & 121 & 169 \\
\hline Ácido fólico (mcg) & 72 & 6 & 9 \\
\hline Vitamina $B_{12}(\mathrm{mcg})$ & 155 & 191 & 123 \\
\hline Ácido pantotênico (mg) & 123 & 67 & 54 \\
\hline Biotina (mcg) & 638 & 113 & 18 \\
\hline Vitamina E (mg ATE) & 76 & 64 & 85 \\
\hline Cálcio (mg) & 41 & 71 & 176 \\
\hline Magnésio (mg) & 50 & 22 & 44 \\
\hline Fósforo (mg) & 38 & 77 & 201 \\
\hline Selênio (mcg) & 47 & 72 & 155 \\
\hline Zinco (mg) & 95 & 60 & 63 \\
\hline Sódio (mg) & 28 & 38 & 135 \\
\hline Potássio (mg) & 15 & 24 & 159 \\
\hline Cobre (mcg) & 94 & 21 & 22 \\
\hline Manganês (mg) & 5 & 2 & 38 \\
\hline Ferro (mg) & 63 & 20 & 31 \\
\hline
\end{tabular}

Os nutrientes destacados em cor cinza foram aqueles que apresentaram média de adequação criticamente baixa em relação ao SI.

SI: valor nutricional do suplemento industrializado comparado com o valor médio de recomendação, utilizando o volume médio estimado; SOA: valor nutricional médio das oito preparações de suplemento artesanal comparado com o valor médio de recomendação, utilizando o volume médio estimado; SOA/SI: valor percentual do SOA comparado como percentual de SI (padrão de referência); Valor médio de recomendação: média dos valores recomendados entre as faixas etárias de 1 a 18 anos; Volume médio estimado: conforme descrito no delineamento - aproximadamente $530 \mathrm{~mL}$.

*Recomendações de energia e proteína ${ }^{15,16}$ que propõem os valores da Recommended Dietary Allowances ${ }^{18}$ e recomendações de micronutrientes - IDR ${ }^{17}$. 
matização dos suplementos no país. A portaria número 32 de 1998 define que os suplementos são alimentos que servem para complementar com nutrientes a dieta diária de uma pessoa saudável, em casos onde a ingestão alimentar seja insuficiente. Os suplementos devem conter no mínimo $25 \%$ e no máximo até $100 \%$ da Ingestão Diária Recomendada de vitaminas e ou minerais, na porção diária indicada pelo fabricante, não podendo substituir os alimentos, nem serem considerados como dieta exclusiva ${ }^{20}$.

Segundo a portaria 40 de 1998 os suplementos não podem ultrapassar os valores indicados como upper level apresentados na Ingestão Diária Recomendada (IDR). Estes valores correspondem à indicação de ingestão máxima diária de micronutrientes ${ }^{21}$.

Considerando esta normatização da ANVISA, notamos que os suplementos artesanais não fornecem quantidades nutricionais acima da quantidade máxima diária recomendada pela IDR, entretanto alguns micronutrientes estão abaixo do corte de $25 \%$ das recomendações como demonstrado na Tabela 2.

\section{Avaliação do sabor}

O sabor do suplemento é um aspecto essencial a ser considerado, principalmente quando estes têm quantidades elevadas de lipídeos, que são úteis para a recuperação do estado nutricional desses pacientes.

Foram 312 avaliações; 167 pacientes experimentaram o suplemento com óleo de soja e 145 com margarina comum sem sal. Globalmente, 254/312 (81,4\%) consideraram o sabor bom, 24/312 (7,7\%) regular e 34/312 (10,9\%) ruim. Quando os suplementos foram comparados para os diferentes tipos de lipídeos, 131/167 (78,4\%) pacientes relataram sabor bom com o óleo e 123/145 $(84,8 \%)$ com margarina: $p=0,15 ; \chi^{2}=2,07$.

O suplemento número 7 (leite com sorvete) foi o único que apresentou tendência à significância estatística $\left(p=0,086 ; \chi^{2}=2,94\right)$. Os pacientes relataram sabor bom com a utilização de óleo (53\%) e margarina (76\%). Os demais suplementos demonstraram diferenças percentuais no relato de sabor bom com os dois tipos de lipídeos, entretanto sem diferenças estatísticas significantes (Figura 1).

A aceitação dos suplementos foi satisfatória, ou seja, mais de $70 \%$ dos que degustaram referiram sabor bom, tanto com a utilização do óleo como com margarina, exceto pela preparação de leite com sorvete quando preparada com óleo (53\%).

Foi verificado baixo percentual (10,9\%) de pacientes que considerou SOA com sabor ruim. A maior porcentagem de rejeição $(21,6 \%)$ esteve associada ao uso do óleo de soja. Quando o SOA foi elaborado com a margarina, menores taxas de rejeição foram encontradas $(15,2 \%)$. Os principais motivos para os relatos de sabor ruim em 34 pacientes foram: sabor muito doce $(2,2 \%)$, odor/sabor ruim ou forte $(2,2 \%)$, cheiro ou sabor de ovo (1,9\%), não gostam de sabor chocolate $(1,3 \%)$ e outros (sabor amargo, pouco doce, sem sabor e consistência ruim).

\section{Discussões acerca das opções de terapia nutricional em crianças e adolescentes com câncer}

A intervenção dietética, isoladamente, não parece capaz de reverter ou prevenir a desnutrição como demonstrado nos estudos de Tyc et al. ${ }^{22} \mathrm{e}$ Bakish et al. ${ }^{23}$, em crianças e adolescentes com câncer, principalmente nas doenças mais avançadas.

A nutrição enteral é definida como o uso de suplementos por via oral ou sonda nasoenteral como medida para prover todos ou parte da energia e nutrientes necessários ${ }^{24}$. Embora em crianças com câncer essa terapia apresente muitas vantagens, incluindo menor risco e custo do que a nutrição parenteral, efetividade na prevenção da depleção nutricional, redução dos episódios febris e de hemoculturas positivas, ela tem recebido pouca atenção no Brasil|25,26-28. 
A suplementação oral por meio de suplementos quer artesanal, quer industrializado, tem sido pouco estudada, provavelmente, porque alguns autores não acreditam na eficácia da via oral como terapia para recuperação nutricional de crianças com câncer ${ }^{25,29}$.

Apesar das evidências quanto às repercussões da desnutrição na criança com câncer, como aumento das toxicidades à quimioterapia, das taxas de complicações infecciosas e da incidência de recaídas, não há definição específica quanto aos benefícios da terapia nutricional nesses pacientes, ainda sendo necessários maiores estudos nesta área ${ }^{30-32}$.

Contudo, é fato que crianças e adolescentes com câncer podem ter importantes alterações da condição nutricional. Por esse motivo, a terapia nutricional pode ser considerada fundamental

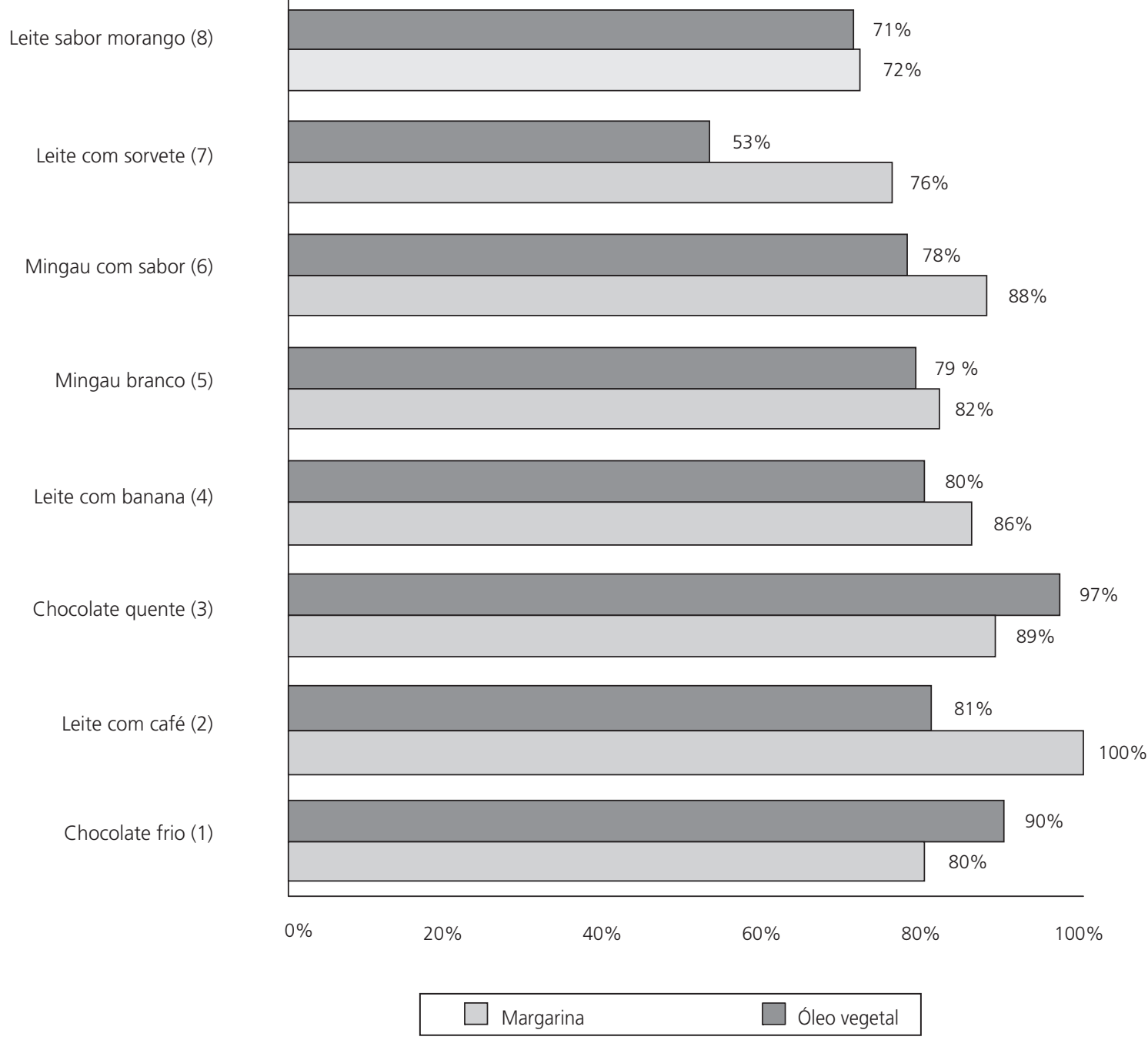

Figura 1. Percentual de pacientes que consideraram o sabor do suplemento bom de acordo com o tipo de suplemento e o tipo de lipídeo utilizado.

Nota: NS= não significante

Leite sabor morango: óleo 118/167 e margarina 104/145; Leite com sorvete: óleo 89/167 e margarina 110/145; mingau com sabor: óleo 130/167 e margarina 128/145; mingau branco: óleo 132/167 e margarina 119/145; leite com banana: óleo 133/167 e margarina 143/145; chocolate quente: óleo 162/167 e margarina 129/145; leite com café: óleo 135/167 e margarina 145/145; chocolate frio: óleo 150/167 e margarina 116/145. 
para estes pacientes, principalmente devido ao prejuízo na ingestão oral, decorrente dos distúrbios gastrintestinais ocasionados pelas toxicidades secundárias ao tratamento $33-38$.

\section{O N CLUS Ã O}

Os resultados encontrados nesse estudo sugerem que o uso de SOA pode ser uma alternativa viável em situações onde não há recursos suficientes para aquisição de suplementos industrializados. Porém, os suplementos desenvolvidos ainda estão sendo testados quanto à quantidade consumida efetivamente por essa população e seu impacto na recuperação do estado nutricional. É importante destacar que medidas como essas devem ser implantadas pelos nutricionistas, principalmente em situações onde não há disponibilidade de recursos para o uso generalizado de SI, o que frequentemente ocorre nos países em desenvolvimento como o Brasil.

\section{COLABORADORES}

A. GARÓFOLO participou da concepção e do desenho do estudo, da análise, da interpretação dos dados e da redação do artigo. F.R. ALVES e M.A.C. REZENDE participaram da análise e da interpretação dos dados. Todos os autores participaram do desenvolvimento dos suplementos.

\section{REFERÊ N CIAS}

1. Petrilli AS, Caran EM, Luisi FA, Barros KVT, Silva NS. Diagnóstico precoce do câncer infantil. Temas de Pediatria-Nestlé. 1992; 50:1-13.

2. Braga PE, Latorre MRDO, Curado MP. Câncer na infância: análise comparativa da incidência, mortalidade e sobrevida em Goiânia (Brasil) e outros países. Cad Saúde Pública. 2002; 18(1):33-44.

3. Lee MLM. Leucemias agudas na infância. Pediatr Mod. 1999; 35:616-21.

4. Tisdale MJ. Cancer cachexia: metabolic alterations and clinical manifestations. Nutrition. 1997; 13(1): 1-7.

5. Ladas EJ, Sacks N, Meacham L, Henry D, Enriquez L, Lowry G, et al. A Multidisciplinary review of nutrition considerations in the pediatric oncology population: a perspective from children's oncology group. Nutr Clin Pract. 2005; 20(4):377-93.

6. Mauer AM,Bugess JB, Donaldson SS, Richard KA, Stallings UA, van Eys J, et al. Special nutrition needs of children with malignancies: a review. JPEN. 1990; 14(3):315-24.

7. Rivadeneira DE, Evoy D, Fahey TJ, Lieberman MD, Daly JM. Nutritional support of the cancer patient. Cancer J Clin. 1998; 48(2):69-80.

8. Brennan, MF. Uncomplicated starvation versus cancer cachexia. Cancer Res. 1977; 37(7 Pt 2): 2359-64.

9. Lawrence W. Effects of cancer on nutrition. Cancer. 1979; 43(S5):2020-9.

10. Inagaki J, Rodriguez $\mathrm{V}$, Bodey GP. Causes of death in cancer patients. Cancer. 1973; 33(2):568-73.

11. Gogos CA, Ginopoulos P, Salsa B, Apostolidou E, Zoumbos NC, Kalfarentzos F. Dietary omega-3 polyunsaturated fatty acids plus vitamin E restore immunodeficiency and prolong survival for severely ill patients with generalized malignancy. A randomized control trial. Cancer. 1998; 82(2): 395-401.

12. Lis CG, Grutsch JF, Vashi PG, Lammersfeld CA. Is serum albumin an independent predictor of survival in patients with breast cancer? JPEN. 2003; 27(1): 10-5.

13. Kyle UG, Pirlich M, Schuetz T, Lochs $H$, Pichard C. Is nutritional depletion by nutritional risk index (NRI) associated with increased length of stay (LOS): a population study. JPEN. 2004; 28(2):99-104.

14. Anção MS, Cuppari L, Tudisco ES, Draibe AS, Sigulem D. Programa de apoio à decisão em nutrição (NUTWIN) versão 2.5. São Paulo: Unifesp; 1995. Departamento de Informática em Saúde Unifesp-EPM.

15. Sacks N, Meek RS. Nutritional support. In: Arthur RA. Supportive care of children with cancer. Current therapy and guidelines from the children's cancer group. $2^{\text {nd }}$ ed. Baltimore: The Johns Hopkins University Press; 1997. p.193.

16. Children's Oncology Group Cancer Control. Algorithm for nutrition intervention and categories of nutritional status in the pediatric oncology patient-references and resources. Washington (DC): COG; 2004.

17. Institute of Medicine. Dietary reference intakes: applications in dietary assessment. Washington (DC): National Academy Press; 2000.

18. National Research Council. Recommended dietary allowances. $10^{\text {th }}$ ed. Washington (DC): National Academy Press; 1989. 
532 A. GARÓFOLO et al.

19. Food and Drug Administration. US Department of Health \& Human Services. [cited 2009 Aug. 20]. Available from: <http:/www.fda.gov/food/ dietarysupplements $>$.

20. Brasil. Ministério da Saúde. Secretaria da Vigilância Sanitária. Portaria n.32 de 13 de janeiro de 1998. Aprovou o regulamento técnico para suplementos vitamínicos e ou minerais. Diário Oficial da União. [acesso 2009 set. 29]. Disponível em: <http://www. anvisa.gov.br>.

21. Brasil. Ministério da Saúde. Secretaria da Vigilância Sanitária. Portaria n.40 de 13 de janeiro de 1998. Aprovou o regulamento que estabelece normas para níveis de dosagens diárias de vitaminas e minerais em medicamentos. Diário Oficial da União. [acesso 2009 set. 29]. Disponível em: <http://www. anvisa.gov.br>.

22. Tyc VL, Vallelunga L, Mahoney S, Snith PS, Mulhern RK. Nutritional and treatment-related characteristics of pediatric oncology patients referred or not referred for nutritional support. Med Pediatr Oncol. 1997; 25(5):379-88.

23. Bakish J, Hargrave D, Tariq N, Laperriere N, Rutka $J T$, Bouffet E. Evaluation of dietetic intervention in children with medulloblastoma or supratentorial primitive neuroectodermal tumors. Cancer. 2003; 98(5):1014-20.

24. Nisim AA, Allins AD. Enteral nutrition support. Nutrition. 2005; 21:109-12.

25. Andrassy RJ, Chwals WJ. Nutritional support of the pediatric oncology patient. Nutrition. 1998; 14(1): 124-9.

26. Sefcick A, Anderton D, Byrne JL, Teahon K, Russell $\mathrm{NH}$. Naso-jejunal feeding in allogeneic bone marrow transplant recipients: results of a pilot study. Bone Marrow Transplantat. 2001; 28(12): 1135-9.

27. American Society for Parenteral and Enteral Nutrition. Board of directors and the clinical guidelines task force. Guidelines for the use of parenteral and enteral nutrition in adult and pediatric patients. JPEN. 2002; 26(1 Suppl):1SA-138SA.
28. Sala A, Wade L, Barr RD. Nutritional support for children with cancer. Ind J Pediatr. 2003; 70(10): 813-6.

29. Pencharz PB. Aggressive oral, enteral or parenteral nutrition: prescriptive decisions in children with cancer. Int J Cancer. 1998; 11:73-5.

30. Donaldson SS, Wesley MN, DeWys WD, Suskind RM, Jaffe N, van Eys J. A study of the nutritional status of pediatric cancer patients. Am J Dis Child. 1981; 135(12):1107-12.

31. Obama M, Cangir A, van Eys J. Nutritional status and anthrcycline cardiotoxicity in children. South Med J. 1983; 76(5):577-8.

32. Taj MM, Pearson AD, Mumford DB, Price L. Effect of nutritional status on the incidence of infection in childhood cancer. Pediatr Hematol Oncol. 1993; 10(3):283-7.

33. Weisdorf SA, Lysne J, Wind D, Haake RJ, Sharp HL, Goldman A, et al. Positive effect of prophylactic total parenteral nutrition on long-term outcome of bone marrow transplantation. Transplantation. 1987; 43(6):833-8.

34. McGeer AJ, Detsky AS, O'Rourke K. Parenteral nutrition in patients receiving cancer chemotherapy. Ann Intern Med. 1989; 110(9):734-6.

35. Harrison LE, Brennan MF. The role of total parenteral nutrition in the patient with cancer. Curr Prob Surg. 1995; 32(10):833-924.

36. Papadoupoulou A. Nutritional considerations in children undergoing bone marrow transplantation. Eur J Clin Nutr. 1998; 52:863-71.

37. Cohen J, Lefor AT. Nutrition support and cancer. Nutrition. 2001; 17(7/8):698-9.

38. Muscaritoli M, Grieco G, Capria S, Iori AP, Fanelli FR. Nutritional and metaboic support in patients undergoing bone marrow transplantation. Am J Clin Nutr. 2002; 75:183-90.

Recebido em: 14/12/2007

Versão final reapresentada em: 3/5/2010 Aprovado em: 6/5/2010 
ANEXO

INGREDIENTES E MODO DE PREPARO DOS SUPLEMENTOS ARTESANAIS

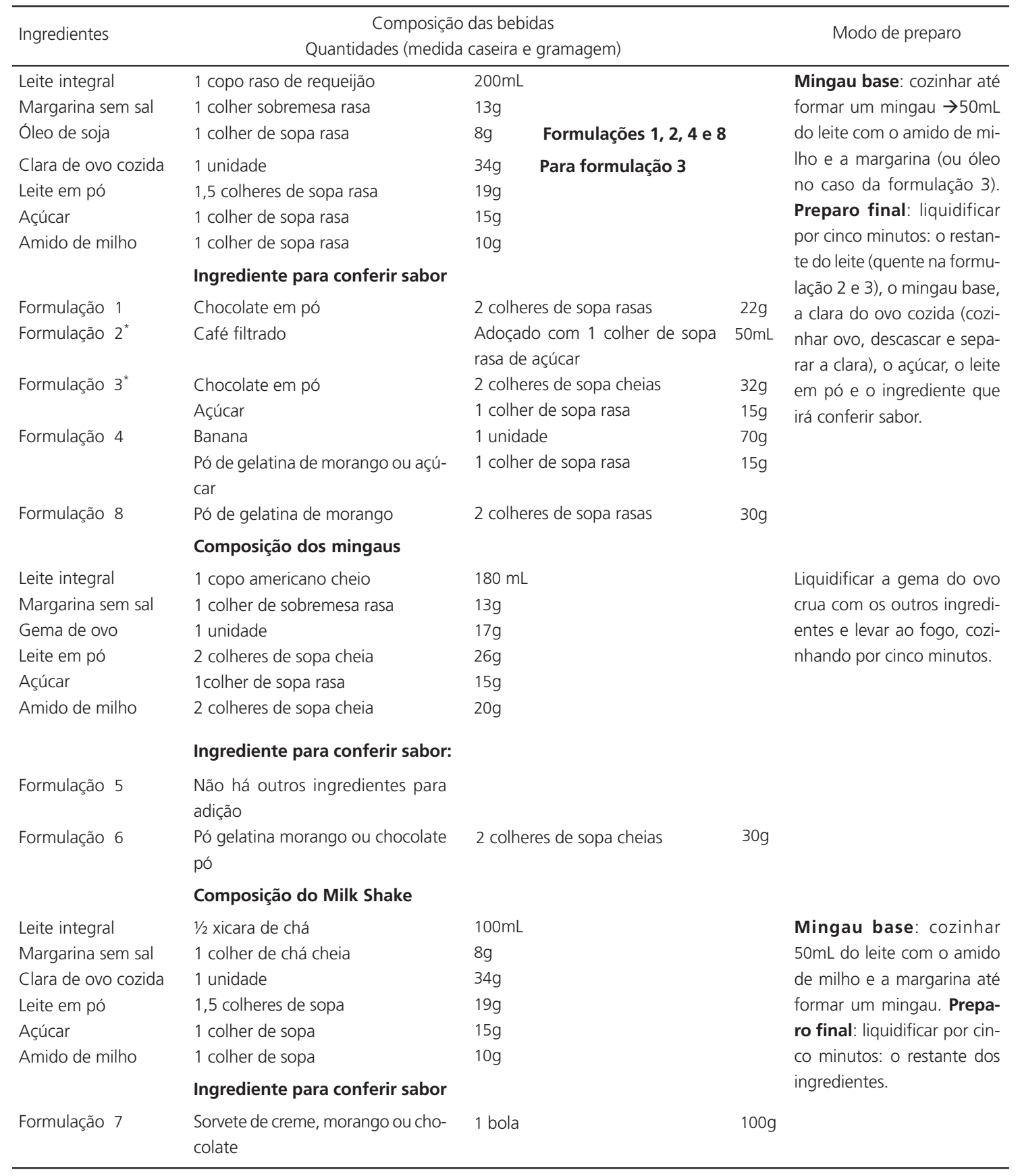

* Servir quente. 